\title{
Vaginal Intraepithelial Neoplasia Detected with Cervical Liquid-Based Cytology: Old Concerns or New Facilities?
}

\author{
Mariana Camezim Beldi*, Maricy Tacla, Marcia Farina Kamilos, Fabiane Sarmanho, \\ Alexandre Ab'Saber, Sheila Siqueira, Venancio Avancini Ferreira Alves, \\ Edmundo C. Baracat, Adhemar Longatto-Filho \\ Hospital das Clinicas of Faculty of Medicine, Sao Paulo State University, Sao Paulo, Brazil \\ Email: * marianabeldi@hotmail.com
}

Received 23 June 2015; accepted 11 September 2015; published 14 September 2015

Copyright (C) 2015 by authors and Scientific Research Publishing Inc.

This work is licensed under the Creative Commons Attribution International License (CC BY). http://creativecommons.org/licenses/by/4.0/

C. (i) Open Access

\begin{abstract}
Background: The detection of vaginal intraepithelial neoplasia (VAIN) in cervical samples is not a common finding. Therefore, we aimed to report VAINs detected in liquid-based cytology (LBC) from women examined at Hospital das Clínicas of Faculty of Medicine, São Paulo State University. Materials and Methods: We evaluated LBC samples from women referred to gynecology examination for different reasons (previous abnormal PapTest, follow up of treated cervical lesion, ecc) and women examined for regular screening proposals, and compared with biopsy diagnoses, including the controversial diagnoses of vaginal intraepithelial neoplasia (VAIN). Results: From 1866 patients, $1423(76.3 \%)$ cases were negative and $443(23.7 \%)$ were positive for any cellular alteration. Age of patients ranged from 12 to 86 years. We detected 25 histologically confirmed VAIN $(1.3 \% p=0.0002$ by Fisher's exact test IC $95 \% 0.0090-0.0198)$ and $1.1 \%$ VAIN $(p=0.0031$ by Fisher's exact test IC 95\% $0.0077-0.0179$ ). Conclusion: The identification of VAIN in routine is feasible; the professionals involved with cytological examination should be aware of these lesions in Pap test samples.
\end{abstract}

\section{Keywords}

Liquid-Based Cytology, Cervical Screening, VAIN, HPV Induced Lesions, Vaginal

\section{Introduction}

Vaginal intraepithelial neoplasia (VAIN) is an infrequent and discreetly known disease, accounting for $<1 \%$ of ${ }^{*}$ Corresponding author.

How to cite this paper: Beldi, M.C., et al. (2015) Vaginal Intraepithelial Neoplasia Detected with Cervical Liquid-Based Cytology: Old Concerns or New Facilities? Open Journal of Obstetrics and Gynecology, 5, 580-587.

http://dx.doi.org/10.4236/ojog.2015.511082 
the lower genital tract [1]. Timonen et al. identified 23 primary vaginal dysplasias among 12,000 patients screened during 1 year. In 1981, Woodruff reported that less than 300 cases of carcinoma in situ of the vagina had been recorded in the literature [2]. Multiple studies have found a number of risk factors for developing VAIN, including a history of previous abnormal Papanicolaou smear, radiotherapy, history of concomitant CIN or cervical cancer, smoking, history of genital warts, immunosuppression, low schooling level and low income [3]. Human papillomavirus (HPV) is the primary initiator of these vaginal lesions and VAIN is classified in a similar manner to CIN [4]. The increasingly widespread application of LBC and improved colposcopic techniques has resulted in the identification of a greater number of low genital tract lesions including VAIN, associated or not with cervical or vulvar lesions [5]. Besides sampling issues during test taking, and slide reading, erroneous results are importantly credited sample preparation and cytological interpretation. Liquid based cytology (LBC) has been developed, in part, to address these issues [6]. Although most studies reported an increased detection of low squamous intraepithelial lesions (LSIL) and decreased inadequacy rates with LBC [7], systematic reviews yielded contradictory results [8].

Once VAIN is a rare disease, we seek to investigate the characteristics of VAIN detected at liquid cytology examination, since we have experienced an increased frequency of VAIN in women enrolled to LBC screening. Also, we evaluated the incidence and risk factors for VAIN among women submitted to gynaecological examination at Hospital das Clínicas of Faculty of Medicine of University São Paulo, São Paulo, Brazil.

\section{Material and Methods}

The Hospital das Clínicas of Faculty of Medicine of São Paulo State University in São Paulo (HC FMUSP) is the biggest Tertiary care center in Latin-America; the Gynecological Care Unit attends around 35,000 women per year. Between August 2010 and November 2011, Women referred to Hospital das Clínicas of Faculty of Medicine of São Paulo State University in São Paulo for gynecological examination due to different reasons (previous abnormal Pap test, follow up of treated cervical lesion, abnormal bleeding and vaginal discharge, breast cancer suspicious, urogenitary prolapses, ecc) were recruit for a cross-sectional study called RODEO STUDY, a multidisciplinary study designed to evaluate the performance of LBC test performance in low-resource settings. Pregnant women and women aged less than 18 years old were excluded. At the first visit, women were enrolled to fill a brief questionnaire that elicited demographic, reproductive and sexual history information. Cytological samples were collected. Women were referred to colposcopy when they presented abnormal Pap smears results (ASC US+). Colposcopic lesions such as thin aceto-white epithelium, fine puntuaction and mosaic (Grade 1 findings) and dense aceto-white epithelium, dense punctuation and mosaic (Grade 2 findings) or suspicious of invasion features (Fragile vessels, Irregular surface, Exophytic lesion, Necrosis, Ulceration tumor or gross neoplasm) were sampled for histological evaluation. For the liquid-based cytology, the slides were prepared and stained with the Papanicolaou stain using Surepath system (Tripath Imaging Inc, Burlington, NC, USA) according to the manufacturer instructions, and screened by skilled cytotechnologists and reviewed by cytopathologists at the Department of Pathology of Hospital das Clínicas Medical Center. The cytological screening was blinded performed to the clinical data. Slides of biopsy specimens were reviewed by the pathologists without knowledge of other clinical or laboratory data. All the women diagnosed with VAIN were follow-up for two years, so this is a prospective branch of the RODEO-STUDY.

\subsection{Preparation of Cytological Samples}

LBC samples were collected with Combi Cervex brush (Rover, Netherland). The brush was integrally inserted into a plastic vial with SurePath liquid-medium (Tripath Imaging Inc., Burlington, NC, USA) and sent to laboratory for semi-automated slide preparation following the manufacturer instructions.

\subsection{Cytological Interpretation}

The slides were classified according to the 2001 Bethesda System. The slide cellularity was considered satisfactory for examination if an estimated minimum of at least 8000/12,000 cells for the conventional smear and 5000 for LBC were observed. The slides with more than $75 \%$ of squamous cells obscured by factors such as inflammatory cells and red blood cells were considered unsatisfactory.

\subsection{Ethics}

The study protocol has been approved by the local Ethic Committee of all participating clinics and all partici- 
pating women gave their agreement to take part of the study by signing the Informed Consent.

\subsection{Statistical Analysis}

Significance was defined as $p \leq 0.05$. Comparisons between proportions were made with the Fischer Exact test, and $95 \%$ confidence intervals (CIs) for corrected estimates were calculated with the use of bootstrap resampling in which the sampling fractions were considered fixed. All analyses were conducted using SPSS software (version 15.0, SPSS Inc., Chicago, IL, USA).

\section{Results}

One thousand eight hundred and sixty six women were included in this study, twenty women denied to be part of the research, so their test results were excluded from the final evaluation. The mean age was 37 years old (range from 19 to 74). Abnormal smears were found in 443 patients (23.7\%). No cases of unsatisfactory slides were detected. From these women, 244 (13\%) presented ASC US, 6 (0.32\%) presented ASC H, 11 (0.58\%) presented AGC and 140 (7.5\%) presented LSIL and 42 (2.25\%) presented HSIL lesions. Histological results were available for 337 patients. We found 7 cases of invasive carcinoma (5 Adenocarcinomas-1 adenosarcoma, 1 adenocarcinoma of the cervix, 3 endometrial adenocarcinoma and 2 squamous cells carcinoma of the cervix). In the ASC US group we were able to confirm by histology 6 cases of CIN 2, one case of CIN 3, 2 cases of VAIN 2, 1 case of VIN 3 and 3 invasive cancers. In the LSIL group we found 10 cases of CIN 2 and 2 cases of VAIN 3. In the HSIL group we were able to find 1 case of squamous invasive carcinoma, 9 cases of CIN 2, 4 cases of CIN 3, 1 case of VAIN 2 and one case of VIN 3 (Table 1).

We detect $25(1.3 \% \mathrm{p}=0.0002$ by Fisher's exact test IC 95\% $0.0090-0.0198)$ histologically confirmed VAIN (Table 2). VAIN was cytologically classified with different types of lesions as showed in Table 2. From these cases, VAIN 1 was found in 20 cases (80\%), VAIN 2 in three cases (11.53\%) and VAIN 3 in two cases (7.7\%). Patients with VAIN were slightest younger than patients with VAIN grade 2 or 3 (36.5 $\times 37.5$ years old). We also found a VAIN incidence of $1.1 \%$ ( $p=0.0031$ by Fisher's exact test IC 95\% $0.0077-0.0179$ ) in our series. One patient was referred to colposcopy because her younger son presents a severe form of recurrent respiratory papillomatosis. Two women have been previously treated for cervical HPV induced lesion (LEEP procedure in 2007 and 2009). We also found VAIN 1 in two patients (8\%) with no cytological abnormalities. CIN was associated with VAIN in five patients (20\%). Three women (21.42\%) were submitted to previous hysterectomy. None of them were smokers or alcoholic. The mean age of their sexual debut was 19 years old (range from 10 to 23 years). Three women (12\%) presented no sexual partner within one year and $84 \%$ patients presented one sexual partner in the last year multiple sexual partners were rarely reported. Regarding parity, three women (12\%) presented more than 5 vaginal deliveries, four (16\%) presented 3 - 4 vaginal deliveries, nine (36\%) presented 1 - 2 deliveries, and nine (36\%) were nulliparous (Table 3). Vulvar lesions were found in five patients (20\%) simultaneously to the vaginal features. The majority of the lesions (76\%) were found in the upper third of the vagina, $16 \%$ (4 cases) in the mid third and two (8\%) in the lower third.

Patient 1 (Figure 1) presented cytological HSIL result. Colposcopy was performed and no cervical but only vaginal atypias were found and the VAIN I histological result was reported. After six months, this patient presented an invasive adenocarcinoma of the cervix. Patient number 4 presented at first, VAIN grade I diagnosis. In the later control (after six months) she presented worsening of the visible vaginal lesion and a new biopsy was taken. It was diagnosed as VAIN 2. Laser vaporization was performed.

\section{Discussion}

This study demonstrated that VAIN is not an infrequent lesions in certain settings. The vagina is the rarest site $(<1 \%)$ of the intraepithelial neoplasia in the lower genital tract. From 1977 to 1986, 0.5\% VAIN frequency was reported among lower genital intraepithelial neoplasm [1]. We found a VAIN frequency of $1.3 \%$ and $1.1 \%$ in our study. We believe that LBC may have contributed in part to the recognition of these lesions in Pap test preparations because its clear background and well-preserved structures are easily recognized. But the cytological classification of these lesions was far from being specific and the morphological presentations were interpreted as squamous lesions of different grades. As far as we know, this is the first report of and systematic VAIN detection by LBC. 
Table 1. Abnormal Pap test and its histological results.

\begin{tabular}{ccccccc}
\hline Cytology & CIN 2 & CIN 3 & Cervix Invasive Carcinoma & VAIN 2 & VAIN 3 & VIN 2 - 3 \\
\hline ASC US & 6 & 2 & 3 & 2 & - & 1 \\
LSIL & 10 & - & - & - & 3 & - \\
HSIL & 9 & 4 & 1 & 1 & - & 1 \\
\hline
\end{tabular}

ASCUS: Atypical Squamous Cells of Undetermined Significance; LSIL: Low Grade Squamous Intraepithelial lesion; HSIL: High Grade Squamous Intraepithelial Lesion; CIN: Cervical Intraepithelial neoplasia; VAIN: Vaginal Intraepithelial neoplasia.

Table 2. Cytological and histopathological results in patients with VAIN diagnoses.

\begin{tabular}{|c|c|c|c|c|c|}
\hline Patient & AGE & $\begin{array}{l}\text { Cytological } \\
\text { result }\end{array}$ & $\begin{array}{l}\text { Histological } \\
\text { result }\end{array}$ & $\begin{array}{c}\text { Previous } \\
\text { Hysterectomy }\end{array}$ & Immunosuppression \\
\hline 1 & 74 & HSIL & VAIN 1 & - & - \\
\hline 2 & 46 & ASC US & VAIN 1 & - & Renal transplant \\
\hline 3 & 58 & LSIL & VAIN 1 & HPV induced lesions & Renal transplant \\
\hline 4 & 23 & ASC US & VAIN 2 & - & HIV+ \\
\hline 5 & 56 & ASC US & VAIN 1 & miomatosis & - \\
\hline 6 & 27 & LSIL & VAIN 1 & & - \\
\hline 7 & 30 & HSIL & VAIN 1 & - & Liver Transplant \\
\hline 8 & 26 & LSIL & VAIN 1 & - & - \\
\hline 9 & 55 & LSIL & VAIN 3 & HPV induced lesions & - \\
\hline 10 & 45 & LSIL & VAIN 1/CIN 1 & - & $\mathrm{HIV}+$ \\
\hline 11 & 19 & LSIL & VAIN 1 & - & Rheumatologic disease \\
\hline 12 & 25 & HSIL & VAIN 1 & - & Liver Transplant \\
\hline 13 & 44 & HSIL & VAIN 2/CIN 2 & - & - \\
\hline 14 & 35 & LSIL & VAIN 1 & - & Rheumatologic disease \\
\hline 15 & 47 & HSIL & VAIN 2 & - & - \\
\hline 16 & 21 & NEGATIVE & VAIN 1 & - & - \\
\hline 17 & 25 & LSIL & VAIN 1/CIN 1 & - & $\begin{array}{l}\text { Rheumatologic } \\
\text { Disease }\end{array}$ \\
\hline 18 & 27 & LSIL & VAIN 1 & - & - \\
\hline 19 & 35 & ASC US & VAIN 2 & - & - \\
\hline 20 & 26 & LSIL & VAIN 1 & - & - \\
\hline 21 & 34 & LSIL & VAIN 1/CIN 1 & - & Rheumatologic disease \\
\hline 22 & 26 & LSIL & VAIN 1/CIN 1 & - & - \\
\hline 23 & 34 & LSIL & VAIN 1/CIN 1 & - & $\begin{array}{l}\text { Rheumatologic } \\
\text { disease }\end{array}$ \\
\hline 24 & 30 & NEGATIVE & VAIN 1 & - & Renal Transplant \\
\hline 25 & 37 & LSIL & VAIN 3 & - & HIV+ \\
\hline
\end{tabular}

ASCUS: Atypical Squamous Cells of Undetermined Significance; LSIL: Low Grade Squamous Intraepithelial lesion; HSIL: High Grade Squamous Intraepithelial Lesion; CIN: Cervical Intraepithelial neoplasia; VAIN: Vaginal Intraepithelial neoplasia.

The improvement in cytological preparations techniques tend to be associated with the better quality of cellular alterations. Actually, there are different liquid-based cytology systems currently available commercially worldwide, but few are approved by American FDA, including SurePath (formerly Autocyte PREP) and LBC system (BD, formerly TriPath Imaging Inc., Burlington, NC, USA). There has been a considerable debate on the merits of LBC compared with conventional cytology, but for developing countries, LBC seems to be more effective than 


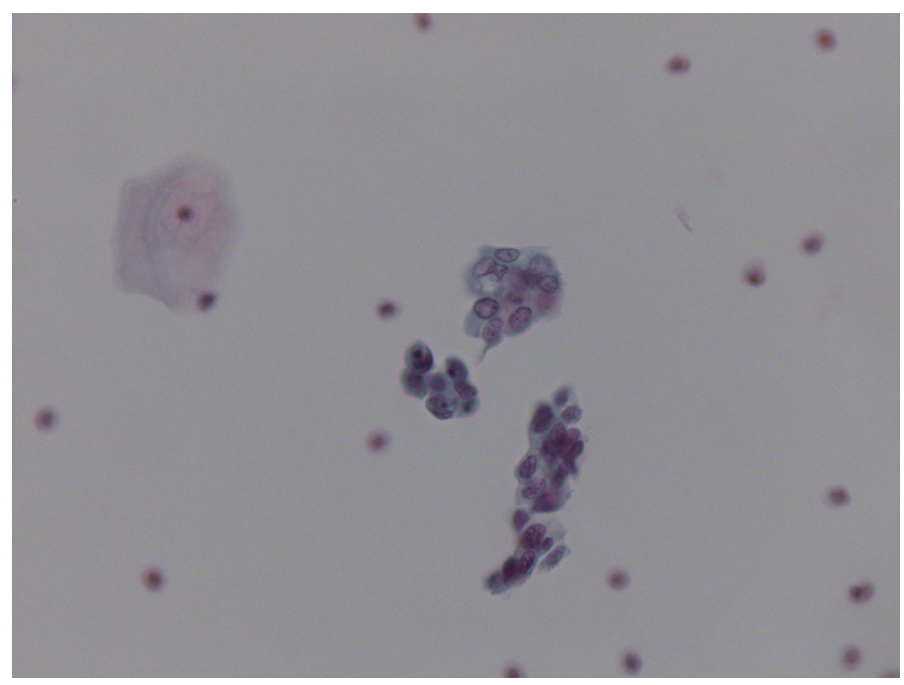

Figure 1. Small group of atypical cells, with prominent dyskariosis, diagnosed as HSIL. (MAGNIFICATION ×40 Papanicoloau stain)

Table 3. Epidemiological aspects of VAIN patients.

\begin{tabular}{|c|c|c|c|}
\hline Patient & Age of sexual debut & Parity & Number of sexual partners (1 year) \\
\hline 1 & 10 & 13 & 0 \\
\hline 2 & 11 & 8 & 1 \\
\hline 3 & 16 & 5 & 1 \\
\hline 4 & 15 & 2 & 1 \\
\hline 5 & 18 & 2 & 1 \\
\hline 6 & 23 & 0 & 0 \\
\hline 7 & 19 & 1 & 1 \\
\hline 8 & 18 & 0 & 1 \\
\hline 9 & 14 & 2 & 0 \\
\hline 10 & 15 & 2 & 1 \\
\hline 11 & 16 & 2 & 1 \\
\hline 12 & 20 & 0 & 1 \\
\hline 13 & 19 & 2 & 1 \\
\hline 14 & 19 & 1 & 1 \\
\hline 15 & 20 & 1 & 1 \\
\hline 16 & 16 & 1 & 1 \\
\hline 17 & 21 & 0 & 4 \\
\hline 18 & 18 & 0 & 1 \\
\hline 19 & 18 & 0 & 1 \\
\hline 20 & 14 & 4 & 1 \\
\hline 21 & 21 & 0 & 1 \\
\hline 22 & 14 & 4 & 1 \\
\hline 23 & 17 & 1 & 1 \\
\hline 24 & 19 & 0 & 1 \\
\hline 25 & 14 & 1 & 1 \\
\hline
\end{tabular}


conventional smears [9]. Arbyn et al. [8] in a systematic review concluded that LBC was neither more sensitive nor more specific for detection of high-grade cervical intraepithelial neoplasia compared with the conventional Pap test. In the other hand, Ronco et al. [10] reported that LBC for primary screening of cervical cancer showed a significant increased sensitivity for cervical intraepithelial neoplasia grade 1 but not for lesions grade 3 or more. Consensually, LBC is associated with a relevant reduction in unsatisfactory samples. The high quality of LBC preparations is certainly out of discussion. Colgan et al. [11] studied 352,680 specimens and they compared the detection and adequacy rates of the SurePath liquid-based cytology system (SP-LBC) with the adequacy and detection rates from a historical conventional smear (CS) group from the same laboratories during the same period of the previous year. They found unsatisfactory rate of $0.24 \%$ and importantly increased detection rate of HSIL by LBC as compared with the detection rate of HSIL by the historical CS group ( $\mathrm{P}=0.0182)$. We have also experienced similar findings since we do not observed unsatisfactory samples in this series. Fremont-Smith et al. [12] evaluated 58,580 SurePath ${ }^{\mathrm{TM}}$ slides and 58,988 historic conventional slides. They found a SurePath detection rate for the clinically important categories of HSIL (64.4\%) and LSIL (107.16\%), and a statistically significant lower proportion of unsatisfactory slides.

Interestingly, Blanks et al. [13] compared the relationship between the cytology result leading to referral to colposcopy and the histology outcome from that referral before and after LBC conversion in English laboratories. The authors examined data from 102 laboratories that converted from conventional cytology to LBC and found before conversion the PPV for CIN2+ of severe dyskaryosis was $88 \%$ and after increased to $90 \%(\mathrm{P}=$ 0.003). For moderate dyskaryosis, the PPV for CIN2+ increased from $70 \%$ to $72 \%(\mathrm{P}=0.06)$. The absolute difference of $18 \%$ between severe and moderate dyskaryosis was therefore the same pre- and post-LBC conversion. The agreement between cytology and histology measured using a weighted Kappa statistic increased from 0.52 to 0.60 after conversion to LBC. Our results corroborate in part this assumption since we detect almost $25 \%$ of lesions in this consecutive series, and fact not observed previously (data not shown). We didn't expect to find so many cases of VAIN in our study. Actually, the cytological alterations that we observed in screening were differently expressed in terms of frequency and cellular characteristics, oscillating from high prominent lesions till discrete morphologic cellular alterations. We can hypothesize if these minimal alterations can be observed or not in conventional smears; based on the previous experience, we can speculate that probably most of VAIN are lost in conventional smear due to the reduced number of altered cells dispersed in dirty background, which certainly limits the efficiency of morphological screening.

The different embryologic origins of the vaginal epithelium are one of the factors underlying the differences in the incidence and, above all, the lesion's evolution. The instability of the metaplastic squamous epithelium of the transformation zone in the cervix appears to favor carcinogenesis [5]. We found VAIN in 3 (12\%) patients submitted to hysterectomy: two were HPV-induced lesions. It is also well established the VAIN association with CIN, VIN or condylomata. We reported four cases (16\%) of VAIN and CIN and five (20\%) condylomata simultaneously with VAIN. Yalcin et al. [14] reported 65.2\% of VAIN lesions associated with CIN, and also Aho [2] reported 50\% of vaginal lesions associated with CIN or VIN. There are few reports of VAIN in LBC samples. Bansal and colleagues [15] reported histopathologic VAIN 2/3 diagnosed from vaginal biopsies in 7 of the 48 patients (14.6\%) with vaginal hrHPV (+) LSIL test results, which was quite similar to our findings.

A plausible reason for this association is that for both sites, cervix and vagina are exposed to the same carcinogenic agents. It is believed that neoplasia develops in vaginal epithelium only in exceptional circumstances [1]. Aho et al. [2] also showed that VAIN lesion could progress; these authors demonstrated that $9 \%$ of the patients had invasive carcinoma after 3 years after VAIN diagnoses. Spontaneous regression is frequent and appears to be more common in cases of VAIN [1]. The question is: does VAIN have the same evolution as CIN? Indracollo et al. [16] in a retrospective study carried out with 2854 patients tried to answer this question. Their results suggest some common features; however, the grade of VAIN may not be strongly linked with the severity of cervical lesion, suggesting a different evolution. Perhaps it is related to the fact that VAIN is not a homogeneous entity, due to HPV infection, as occurs in CIN. Only 36\% of our VAIN, 1 case was detected in younger patients ( $>30$ years old). This is an important finding, in view of the fact that these features are not just signs of a transient infection. The upper third region of the vagina was the most frequently involved site. Yalcin [14] reported the upper third section was involved in 95.8\% and Indracollo et al. [15] found 51\% - 98\% of the patients with lesions in the upper third.

Prior hysterectomy for cervical neoplasia, history of simultaneous presence of CIN, previous pelvic radiation, immunosuppression and smoking are among risk factors for VAIN [17]. Also, it is well known that patients 
submitted to transplant who are undergoing immunosuppressive therapy are found to be at increased risk of developing intraepithelial neoplasia. An increased incidence of CIN has been described in patients who have received renal transplants and the transient immunosuppression which occurs in pregnancy is associated with an increased incidence of genital warts and CIN lesions and a higher prevalence of HPV in cervical smears [18]. The incidence of cervical as well as anal precursor lesions and cancer is markedly higher in HIV-positive men and women compared with HIV-negative men and women [19]. Interaction between HPV and HIV has been demonstrated in vitro with the tat-I protein of HIV capable of enhancing E2 dependent HPV 16 transcription: this supposedly provides a potential mechanism for interaction between these agents and suggests that the interaction may occur in two separate ways, that is, by immunosuppression and by direct alteration of gene transcription [18]. Massad et al. [20] estimated the prevalence, incidence, and clearance of abnormal vaginal cytology and vaginal intraepithelial neoplasia (VAIN) in human immunodeficiency virus (HIV)-seropositive women. They evaluated 335 HIV-seropositive and 75 HIV-seronegative women with prior hysterectomy. The incidence of VAIN 2 or worse was 0.2 for HIV-seropositive and HIV-seronegative women. They concluded that although more common than in HIV-seronegative women, VAIN 2 or worse and especially vaginal cancers were infrequent. Our series support this conjecture since from 25 patients with VAIN, 13 have concomitant history of immunosuppression, 3 of them HIV+, 5 due to organ transplantation and 5 because of rheumatoid diseases.

An important limitation regarding our findings is the fact that all women came from HCFMUSP with a particular gynecological issue, this population is somehow different from an usual screening one. In fact, our hospital is a reference for genital lesions treatment and possibly the high frequency of VAIN is a consequence of this status. However, even considering this conjecture as a valid hypothesis, we didn't expect and never experience so many cases of VAIN in such short period of time. Despite the fact that vaginal cancer is the rarest type of cancer among women, Aho et al. [2] have shown us that VAIN does progress to invasive forms. So, we do believe that any kind of improvement must be seen with enthusiasm.

In conclusion, our work revealed additional improvements with the adoption of LBC in our routine of gynecological examination, even ameliorating the detection of VAIN, a rare condition.

\section{References}

[1] Sillman, F.H., Fruchter, R.G., Chen, Y.S., et al. (1997) Vaginal Intraepithelial Neoplasia: Risk Factors for Persistence, Recurrence, and Invasion and Its Management. American Journal of Obstetrics \& Gynecology, 176, 93-99. http://dx.doi.org/10.1016/S0002-9378(97)80018-X

[2] Aho, M., Vesterinen, E., Meyer, B., et al. (1991) Natural History of Vaginal Intraepithelial Neoplasia. Cancer, 68, 195-197. http://dx.doi.org/10.1002/1097-0142(19910701)68:1<195::AID-CNCR2820680135>3.0.CO;2-L

[3] Indermaur, M.D., Martino, M.A., Fiorica, J.V., et al. (2005) Upper Vaginectomy for the Treatment of Vaginal Intraepithelial Neoplasia. American Journal of Obstetrics \& Gynecology, 193, 577-580, Discussion 580-581. http://dx.doi.org/10.1016/j.ajog.2005.03.055

[4] Boonlikit, S. and Noinual, N. (2010) Vaginal Intraepithelial Neoplasia: A Retrospective Analysis of Clinical Features and Colpohistology. Journal of Obstetrics and Gynaecology Research, 36, 94-100. http://dx.doi.org/10.1111/j.1447-0756.2009.01108.x

[5] Minucci, D., et al. (1995) Epidemiological Aspects of Vaginal Intraepithelial Neoplasia (VAIN). Clinical \& Experimental Obstetrics \& Gynecology, 22, 36-42.

[6] Siebers, A.G., et al. (2008) Cytologic Detection of Cervical Abnormalities Using Liquid-Based Compared with Conventional Cytology: A Randomized Controlled Trial. Obstetrics \& Gynecology, 112, 1327-1334. http://dx.doi.org/10.1097/AOG.0b013e31818c2b20

[7] Sykes, P.H., Harker, D.Y., Miller, A., et al. (2008) A Randomised Comparison of Sure Path Liquid-Based Cytology and Conventional Smear Cytology in a Colposcopy Clinic Setting. BJOG, 115, 1375-1381. http://dx.doi.org/10.1111/j.1471-0528.2008.01865.x

[8] Arbyn, M., et al. (2008) Liquid Compared with Conventional Cervical Cytology: A Systematic Review and Meta-Analysis. Obstetrics \& Gynecology, 111, 167-77. http://dx.doi.org/10.1097/01.AOG.0000296488.85807.b3

[9] Scapulatempo, C., Fregnani, J.H, Campacci, N., Possati-Resende, J.C., Longatto-Filho, A. and Rodeo Study Team (2013) The Significance of Augmented High-Grade Squamous Intraepithelial Lesion Detection on Pap Test Examination: Partial Results from the RODEO Study Team. Acta Cytologica, 57, 489-494. http://dx.doi.org/10.1159/000351789

[10] Ronco, G., Cuzick, J., Pierotti, P., et al. (2007) Accuracy of Liquid Based versus Conventional Cytology: Overall Re- 
sults of New Technologies for Cervical Cancer Screening: Randomised Controlled Trial. BMJ, 335, 28. http://dx.doi.org/10.1136/bmj.39196.740995.BE

[11] Colgan, T.J., McLachlin, C.M., Cotterchio, M., Howlett, R., Seidenfeld, A.M. and Mai, V.M. (2004) Results of the Implementation of Liquid-Based Cytology-SurePath in the Ontario Screening Program. Cancer Cytopathology, 102, 362-367. http://dx.doi.org/10.1002/cncr.20656

[12] Fremont-Smith, M., Marino, J., Griffin, B., Spencer, L. and Bolick, D. (2004) Comparison of the SurePath ${ }^{\mathrm{TM}}$ Liquid- $^{2}$ Based Papanicolaou Smear with the Conventional Papanicolaou Smear in a Multisite Direct-to-Vial Study. Cancer Cytopathology, 102, 269-279. http://dx.doi.org/10.1002/cncr.20599

[13] Blanks, R.G. and Kelly, R.S. (2010) Comparison of Cytology and Histology Results in English Cervical Screening Laboratories before and after Liquid-Based Cytology Conversion: Do the Data Provide Evidence for a Single Category of High-Grade Dyskaryosis? Cytopathology, 21, 368-373. http://dx.doi.org/10.1111/j.1365-2303.2010.00808.x

[14] Yalcin, O.T., Rutherford, T.J., Chambers, S.K., Chambers, J.T. and Schwartz, P.E. (2003) Vaginal Intraepithelial Neoplasia: Treatment by Carbon Dioxide Laser and Risk Factors for Failure. European Journal of Obstetrics \& Gynecology and Reproductive Biology, 106, 64-68. http://dx.doi.org/10.1016/S0301-2115(02)00209-9

[15] Bansal, M., Austin, R.M. and Zhao, C.Q. (2011) Correlation of Histopathologic Follow-Up Findings with Vaginal Human Papillomavirus and Low-Grade Squamous Intraepithelial Lesion Papanicolaou Test Results. Archives of Pathology \& Laboratory Medicine, 135, 1545-1549. http://dx.doi.org/10.5858/arpa.2010-0658-OA

[16] Indraccolo, U., Chiocci, L. and Baldoni, A. (2008) Does Vaginal Intraepithelial Neoplasia Have the Same Evolution as Cervical Intraepithelial Neoplasia? European Journal of Gynaecological Oncology, 29, 371-373.

[17] Frega, A., French, D., Piazze, J., Cerekja, A., Vetrano, G. and Moscarini, M. (2007) Prediction of Persistent Vaginal Intraepithelial Neoplasia in Previously Hysterectomized Women by High-Risk HPV DNA Detection. Cancer Letters, 249, 235-241. http://dx.doi.org/10.1016/j.canlet.2006.09.003

[18] Herrington, C.S. (1995) Human Papillomaviruses and Cervical Neoplasia. II. Interaction of HPV with Other Factors. Journal of Clinical Pathology, 48, 1-6. http://dx.doi.org/10.1136/jcp.48.1.1

[19] van der Burg, S.H. and Palefsky, J.M. (2009) Human Immunodeficiency Virus and Human Papilloma Virus—Why HPV-Induced Lesions Do Not Spontaneously Resolve and Why Therapeutic Vaccination Can Be Successful. Journal of Translational Medicine, 7, 108. http://dx.doi.org/10.1186/1479-5876-7-108

[20] Massad, L.S., Xie, X.H., Greenblatt, R.M., Minkoff, H., Sanchez-Keeland, L., Watts, D.H., et al. (2012) Effect of Human Immunodeficiency Virus Infection on the Prevalence and Incidence of Vaginal Intraepithelial Neoplasia. Obstetrics \& Gynecology, 119, 582-589. http://dx.doi.org/10.1097/AOG.0b013e318244ee3d 